\title{
Effect of Endurance Training and Testosterone Treatment on the Fluorescence Spectra of Rat Serum
}

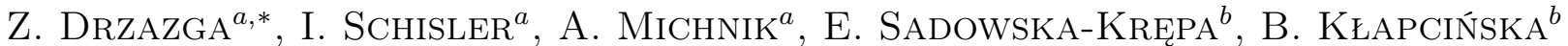 \\ AND J. GIBIŃSKA ${ }^{a}$ \\ ${ }^{a}$ Department of Medical Physics, Chełkowski's Institute of Physics, University of Silesia, \\ Uniwersytecka 4, 40-007 Katowice, Poland \\ ${ }^{b}$ Department of Biochemistry, The Jerzy Kukuczka Academy of Physical Education, \\ Mikołowska 72a, 40-065 Katowice, Poland \\ (Received July 30, 2015)
}

\begin{abstract}
The paper presents an investigation into the effects of endurance training and testosterone enanthate (TE) treatment on rat blood serum by fluorescence spectroscopy. Blood serum was collected from adolescent male Wistar rats randomized between the untrained controls and trained animals, either steroid untreated or receiving I.M. injections ( 8 or $80 \mathrm{mg} / \mathrm{kg}_{\mathrm{BW}}$ ) of testosterone enanthate. Fluorescence measurements were performed for undiluted or 20- and 500-fold diluted serum and analysed separately in the ultraviolet and visible light regions. In both regions the impact of the TE administration was more pronounced than the effect of endurance training. Moreover, in the visible region of spectrum, originated from a number of fluorescent metabolites, the influence of studied factors was more noticeable. The opposite effect of endurance training and administration of TE on serum emission was found for a lower $\left(8 \mathrm{mg} / \mathrm{kg}_{\mathrm{BW}}\right)$ dose of testosterone, whereas a high-dose $\mathrm{TE}(80 \mathrm{mg} / \mathrm{kg} \mathrm{BW})$ treatment resulted in a return of fluorescence intensity.
\end{abstract}

DOI: 10.12693/APhysPolA.129.15

PACS: 33.50.Dq, 87.10.Vg, 87.64.-t, 87.64.kv

\section{Introduction}

Regular exercise training, recommended to improve athletic performance, is often combined with anabolicandrogen steroid (AAS) treatment, especially in young athletes. In the short term, the combination of regular physical activity and administration of AAS doses can increase strength and muscle mass and affect erythropoiesis and blood hemoglobin concentrations [1]. The relationship between the trainability of individuals subjected to physical exercise and the levels of circulating testosterone was discussed by Cadore and Kruel [2]. However, AAS abuse may cause a number of side effects, such as an increment in aggressive behaviour and enhancement of cardiovascular risk factors including elevated blood pressure and reduced high density lipoprotein in the blood serum $[3,4]$. Prolonged use of AAS in relatively high doses lead to hypogonadism, with decreased serum concentrations of luteinizing hormone, follicle stimulation hormone and endogenous testosterone [1]. Recently the possibility of calorimetric monitoring of the effect of endurance training and administration of testosterone doses on the process of rat serum thermal denaturation has been pointed [5].

However, to our knowledge, no systematic study has been undertaken on the effects of endurance training and testosterone treatment on the fluorescence spectra of blood serum. Alterations in the fluorescence spectra of endogenous and exogenous biological chromophores are

*corresponding author; e-mail: zofia.k.drzazga@gmail.com related to the environment and the current metabolic status of the tissue, which may vary with the transition from the normal to the pathological state $[6,7]$. The detection of emission signals has been employed in medicine as an effective and non-invasive probe of biochemical and biomorphological alterations in precancerous tissue. Many efforts have been made to optimize the detection and imaging of diseased tissues, exploring the diagnostic potential of exogenous fluorophores developed for photodynamic diagnosis and therapy [8]. Fluorescence spectroscopy may also give valuable information in non-cancerous cases, for example, fluorescence spectroscopy was applied as a tool for bone development monitoring in new-born rats $[9,10]$.

Serum contains $60-80 \mathrm{~g}$ of protein $/ 1$ and various small molecules, including salts, lipids, amino acids and sugars. All fractions of serum proteins (albumin, alphaglobulins, beta-globulins and gamma globulins) emit the specific fluorescence spectrum in the ultraviolet region, mainly due to the presence of three aromatic amino acids: phenylalanine (Phe), tyrosine (Tyr) and tryptophan (Trp) in their structures. It was demonstrated that the patterns of fluorescence in diluted serum samples might provide information about the health status of the individual [11-14]. Characteristic differences in the Trp fluorescence spectra of sera, as a way of getting information about neoplastic diseases, were proposed by Leiner et al. [12] and Hubmann [13]. Fluorescence spectroscopy in combination with advanced multivariate data analysis of serum samples to discriminate healthy females from breast cancer patients was presented by Nørgaard et al. [14]. 
This paper presents an investigation in an animal model regarding the usefulness of fluorescence spectroscopy of serum samples for testing the effect of endurance training alone or combined with administration of testosterone enanthate (TE). For this purpose, the emission spectra of serum derived from sedentary or endurance-trained rats subjected to treatment with two different doses of exogenous steroid were studied.

\section{Experimental}

Thirty-six adolescent male Wistar rats from the Mossakowski Medical Research Centre (Warsaw, Poland) were used for the study. The study protocol was approved by the First Warsaw Ethical Committee for Animal Experiments of the Polish Academy of Sciences, Warsaw, Poland (Certificate of approval No. 251).

The selected rats were randomized among the following groups: untrained controls (UTr, $N=6$ ), untrained rats receiving $8 \mathrm{mg} / \mathrm{kg}_{\mathrm{BW}}(\mathrm{UTr}+\mathrm{TE} 8, N=6$ ) or $80 \mathrm{mg} / \mathrm{kg}_{\mathrm{BW}}(\mathrm{UTr}+\mathrm{TE} 80, N=6)$ of testosterone enanthate (TE) weekly, endurance-trained steroid-untreated rats ( $\operatorname{Tr}, N=6$ ), and endurance-trained rats receiving $8 \mathrm{mg} / \mathrm{kg}_{\mathrm{BW}}(\mathrm{Tr}+\mathrm{TE} 8, N=6)$ or $80 \mathrm{mg} / \mathrm{kg}_{\mathrm{BW}}$ of $\mathrm{TE}$ weekly $(\mathrm{Tr}+\mathrm{TE} 80, N=6)$. A stock TE solution was diluted with sesame oil as needed and injected intramuscularly once a week for six weeks, alternately into the right and left hind limbs. TE-untreated rats were given the same volume of the oil according to the same schedule. It should be noted that the lower TE dosage ( $\left.8 \mathrm{mg} / \mathrm{kg}_{\mathrm{BW}}\right)$ approximated well the AAS dosage typically used by athletes while the higher TE dosage roughly matched the maximum reported AAS dosage [15, 16].

The rats scheduled for endurance training were exercised on a rodent treadmill five days a week for six weeks, as described previously [15]. Two days after the completion of the training, the rats were killed by decapitation, and the trunk blood was collected. The collected blood was allowed to clot at room temperature and spun to obtain serum for the assessment of total testosterone (TT) and free fatty acids (FFAs) using commercially available diagnostic kits KIPI19000 (DIASource Immunoassays S.A) and Randox FA 115, respectively.

The samples of rat sera were stored at $-80^{\circ} \mathrm{C}$ until the fluorescence was measured. The fluorescence study was conducted at room temperature on the blood serum collected from six rats for each of the six groups mentioned. Fluorescence measurements of undiluted and diluted serum samples were performed. For acquisition of the data for fluorescence spectra in the visible region, the sera were diluted 1:20 with degassed redistilled water (DDW), whereas for the analysis of spectra in the ultraviolet region, a 1:500 dilution was applied. The $\mathrm{pH}$ of the diluted samples was within the range of 6.5 to 7 and 7-7.5 for the 1:500 and 1:20 diluted samples, respectively. Complete excitation emission matrices (EMMs) involving the fluorescence intensity were registered using a spectrofluorometer Hitachi F-2500 with software FLSolutions. The following instrumental settings were used: the slit widths for experiments were $2.5 \mathrm{~nm}$ (excitation) and $5.0 \mathrm{~nm}$ (emission). The emission spectra of undiluted samples were recorded with $5.0 \mathrm{~nm}$ step at the excitation wavelengths from 240 to $420 \mathrm{~nm}$ while emission spectra of diluted samples in the both ultraviolet and visible region, separately, could be scanned with $1.0 \mathrm{~nm}$ step.

Statistical analysis of the results was performed with Statistica 10 using ANOVA or the nonparametric U-Mann Whitney test and the post hoc NIR test. The Shapiro-Wilk and the Levene tests were used to check the normality of the distributions and the homogeneity of variance, respectively. Differences with a $p<0.05$ were regarded as significant.

\section{Results and discussion}

\subsection{Mapping of rat serum fluorescence}

A sample 3D presentation of total fluorescence of undiluted serum is shown in Fig. 1a.

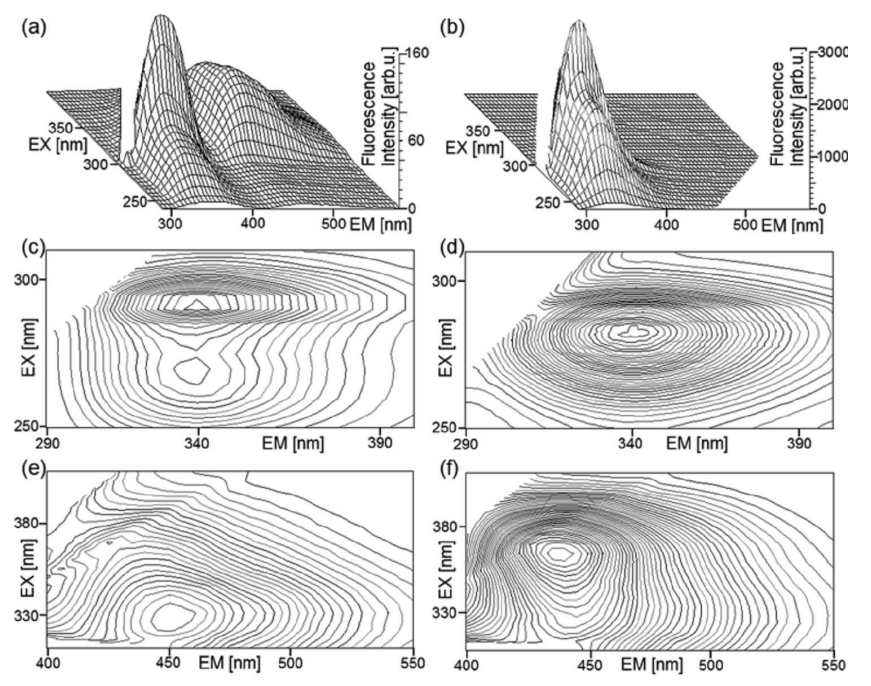

Fig. 1. Sample presentations of excitation emission matrix: (a) raw serum from the untrained control group (UTr), (b) the same sample diluted 1:20, (c, d) the ultraviolet region for serum from group ( $\mathrm{Tr}+\mathrm{TE} 8)$ at dilutions of 1:20 and 1:500, respectively, $(\mathrm{e}, \mathrm{f})$ the visible region for serum from UTr groups and $\mathrm{Tr}+\mathrm{TE} 8$, respectively (dilution 1:20).

Two well-separated parts of the emission in the ultraviolet and visible regions have been detected for all studied serum samples. Both peaks are broad and reveal the complex nature of autofluorescence in serum. For an excitation with ultraviolet radiation wavelengths, a strong emission peak with a maximum at approximately $345 \mathrm{~nm}$ (ex/em 305/345 nm) with the marked elongated shoulder towards the lower wavelengths excitation was observed in all studied samples. This emission may be identified as fluorescence resulting from aromatic amino acids emission bands originating from numerous serum proteins. The fundamentals of protein fluorescence have been summarized by Lakowicz [17]. The second part of the fluorescence occurring in the visible region is attributed to 
closely spaced overlapping emissions generated by a number of low molecular mass fluorescent compounds such as metabolic coenzymes (NAD $(\mathrm{P}) \mathrm{H}$, flavins) or the Schiff base of pyridoxal phosphate with amino acids according to the literature $[11,14]$ assuming that there are analogies in the fluorescence of human and rat serum (despite some proteins that are unique to rats [18]).

However, the fluorescence of complex mixtures may not be additive due to quenching phenomena and interactions with the molecular environment of the fluorophores. Therefore, an analysis has been conducted on the spectra of appropriately diluted samples of rat serum, assuming that the overall signal of fluorescence could be expressed as the sum of the fluorescence contributions from each of the fluorophores.

A dilution of serum causes an increase in fluorescence in the ultraviolet region and a drop in fluorescence in the visible region (Fig. 1b). Therefore, the analysis of the results was conducted in both regions separately, as was previously proposed by Wolfbeis and Leiner [11].

A representative ultraviolet region of the fluorescence topogram of the serum diluted 1:20 and 1:500 is shown in Fig. 1c and d, respectively. The dominating peak originating from the fluorescence of Trp residue (ex/em 290/335 $\mathrm{nm}$ ) and the second smaller maximum, which can be attributed to the fluorescence of Tyr (ex/em 270/335 nm), are visible in two-dimensional contour plots forming an elongated ellipsoid for the 1:20 dilution (Fig. 1c). With an increasing dilution of serum, the recorded patterns become more symmetrical due to the disappearance of the Tyr emission. A loss of Tyr fluorescence may be interpreted in terms of efficient Förster resonance energy transfer (FRET) from Tyr to Trp residues [15] due to the partial overlap of the Tyr emission with the absorption spectrum of Trp. For serum samples diluted 1:500, the contour plots (Fig. 1d) indicate only the maximum of the Trp emission (ex/em 282/339 nm).

Figure 1e-f presents characteristic EEMs registered in the visible region for the studied rat serum samples. It should be noted that the two-dimensional contour plots of fluorescence differ from one another. The shift of maximum fluorescence between 435 and $455 \mathrm{~nm}$ in the excitation range of 325-365 nm dependent of the studied sample was registered. An asymmetric shape of the contour lines of the emission (Fig. 1f) was observed for the serum from the endurance-trained rats and the endurancetrained and steroid-treated ( $\mathrm{TE}=8 \mathrm{mg} / \mathrm{kg}_{\mathrm{BW}}$ ) more often (up to $40 \%$ ) than for the other groups, especially the sedentary group, which showed less asymmetric patterns (Fig. 1e). Such attributes of the emission spectra may be connected with the unstable endogenous fluorophores in the rat serum. Thus, fluorescence profiles under excitation wavelengths of 325 and $365 \mathrm{~nm}$ have been chosen for better insight into the spectral differences (Fig. 2a).

The shape of the curves at ex. $365 \mathrm{~nm}$ is more complex than those at $325 \mathrm{~nm}$, and their intensities are lower under excitation $365 \mathrm{~nm}$ than $325 \mathrm{~nm}$ for all trained group analogues (treated with the same dose
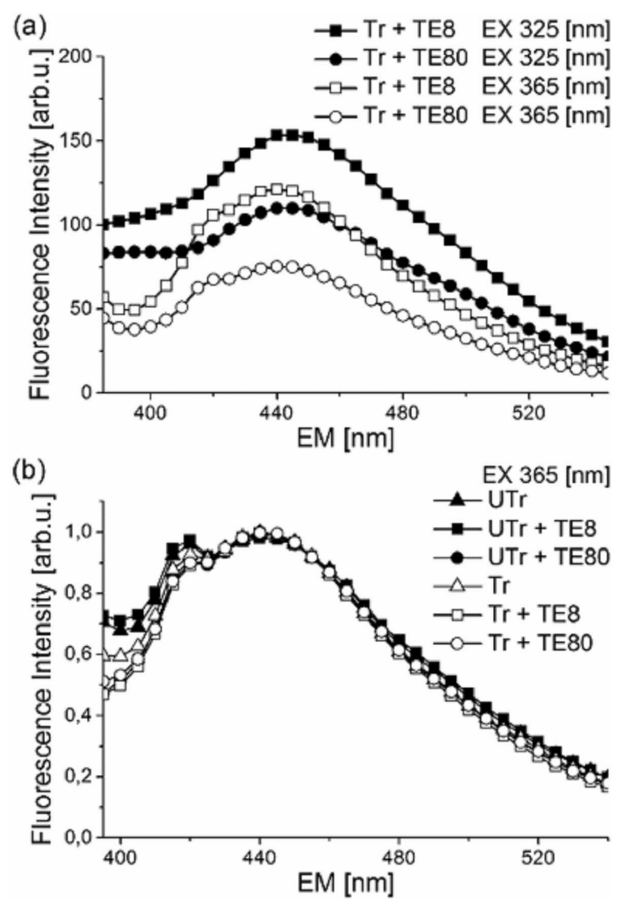

Fig. 2. The average emission spectra of rat sera: (a) ex. 325 and $365 \mathrm{~nm}$ (dilution 1:20), (b) ex. $365 \mathrm{~nm}$ after normalization (dilution 1:20).

of TE). The normalization of averaged spectra at $365 \mathrm{~nm}$ (Fig. 2b) reveals contributions from pyridoxic acid lactone (ex/em $365 / 420 \mathrm{~nm}$ ) for all trained groups of rats and clearly indicates a stable maximum of fluorescence at $440 \mathrm{~nm}$, which is thought to be characteristic for $\mathrm{NAD}(\mathrm{P}) \mathrm{H}$ and also for pteridine derivatives. However, the concentration of these species in serum and thereby their emission are very low; therefore, the fluorescence of advanced glycation end products (AGEs) having similar spectra was suggested by Münch [19]. Recently, studies on AGEs of human serum albumin by means of circular dichroism, fluorescence spectroscopy and differential scanning calorimetry have been reported by Bohlooli et al. [20]. The fluorescence spectra at 325 and $365 \mathrm{~nm}$ obtained in our study seem to be similar to those from glycated human serum. This may suggest an effect of AGEs on emissions in visible region, but a hypothesis that endurance training and testosterone treatment may affect AGE levels requires further study.

\subsection{Effect of endurance training and testosterone treatment on serum fluorescence}

Differences between emission curves of serum samples of steroid-treated rats and untreated rats in the ultraviolet region are shown in Fig. 3. Some increase of Trp emission of serum from trained and sedentary rats treated with lower dose of testosterone (TE8), but the high dose testosterone treatment (TE80) reverses this effect. The effect of TE dose is more marked in trained than in sedentary rats, but unfortunately, the observed changes in fluorescence are not statistically significant, 
probably due to large inter-individual variability. The effect of testosterone dosage may be connected with modification of fluorescence of albumin, the protein involved in transport of serum endogenous steroids, as well as transport of fatty acids in the circulation [21].

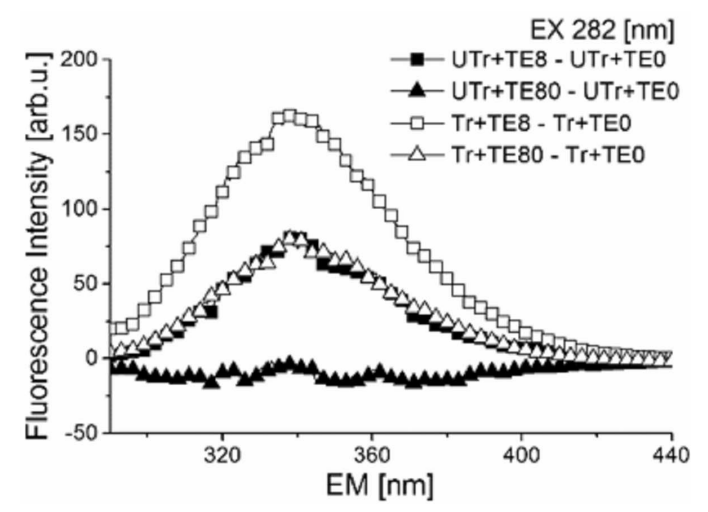

Fig. 3. Differences between average emission curves of serum samples of steroid-treated rats and untreated rats in the ultraviolet region (dilution 1:500).
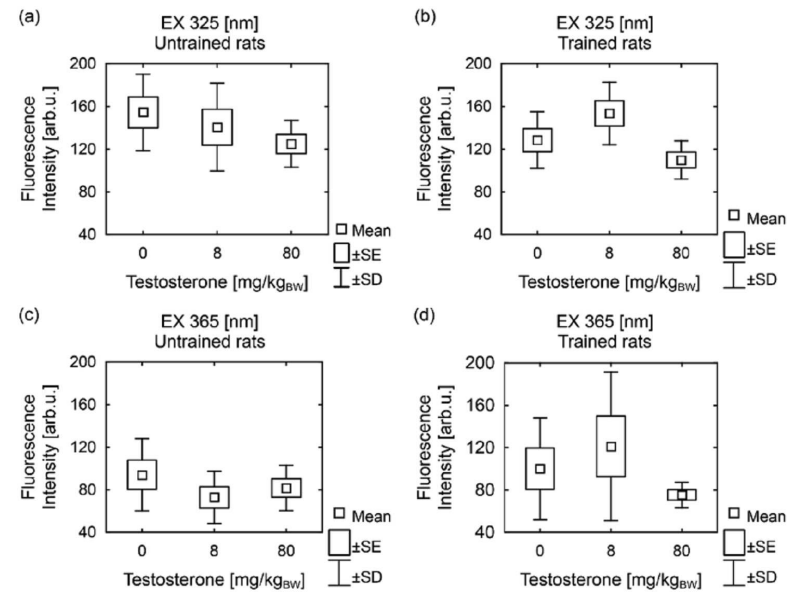

Fig. 4. Average values of emission maximum of diluted 1:20 serum as a function of testosterone dose at ex. $325 \mathrm{~nm}(\mathrm{a}, \mathrm{b})$ and at ex. $365 \mathrm{~nm}$ (c, d) for the untrained and endurance-trained groups of rats, respectively. At ex. $325 \mathrm{~nm}$ significant changes were obtained between $\mathrm{UTr}+\mathrm{TE} 0$ and $\operatorname{Tr}+\mathrm{TE} 80$ as well as Tr+TE8 and $\mathrm{Tr}+\mathrm{T} 80$ groups (Test NIR) and at ex. $365 \mathrm{~nm}$ between UTr+TE8 and Tr+TE8 groups (Test U MannWhitney).

Serum fluorescence spectra in the visible region are changing substantially (Figs. 1e,f and 2), although the excitation and emission ranges of serum fluorescent species overlap to a large extent, so there are no separate emission peaks in this region. Therefore, a special focus was put on changes in the maximum fluorescence intensities under specific excitation wavelengths $(325 \mathrm{~nm}$ and $365 \mathrm{~nm})$ as a function of the experimental conditions, i.e., sedentary/trained and steroid treated/untreated groups (Fig. 4).
Serum emission spectra at $325 \mathrm{~nm}$ show the marked tendency to a systematic decrease with increasing dose of TE in samples collected from untrained (Fig. 4a) but not endurance-trained animals.

The administration of a lower dose of TE $\left(8 \mathrm{mg} / \mathrm{kg}_{\mathrm{BW}}\right)$ in trained animals caused some increase in serum fluorescence (Fig. 4b), but the opposite effect was observed in animals given a large steroid dose $\left(80 \mathrm{mg} / \mathrm{kg}_{\mathrm{BW}}\right)$. Statistically significant differences were found between the $\operatorname{Tr}+\mathrm{TE} 8$ and $\operatorname{Tr}+\operatorname{TE} 80(p=0.02)$, as well as between the UTr and $\operatorname{Tr}+\mathrm{TE} 80(p=0.04)$ groups. Moreover, it seems that training alone may somehow contribute to a decrease in fluorescence at $325 \mathrm{~nm}$ in serum from steroiduntreated rats, but the differences between UTr and $\operatorname{Tr}$ groups have not reached statistical significance.

Among observed differences in maximum emission at $365 \mathrm{~nm}$, only those observed in serum spectra from $\mathrm{UTr}+\mathrm{TE} 8$ and $\mathrm{Tr}+\mathrm{TE} 8$ groups of animals were statistically significant (Fig. 4c and d). This may imply that endurance training potentiates the effect of steroid treatment on maximum emission at $365 \mathrm{~nm}$; however, treatment with a higher dose of $\mathrm{TE}\left(80 \mathrm{mg} / \mathrm{kg}_{\mathrm{BW}}\right)$ reverses the effect of endurance training. The possibility of the antagonistic effects of testosterone and physical activity on muscle fiber diameter in rats was reported by Hodosy et al. [22]. Similar negative effect of a high-dose testosterone treatment on myocardial antioxidant defences was found in adolescent male rats [15].

Moreover, an attempt was made to find a relationship between the intensity of autofluorescence and biochemical parameters in sedentary and endurance-trained rats treated with different doses of testosterone. Statistically significant differences were observed for serum fluorescence in the visible region only. The negative moderate correlation between the fluorescence intensity at an excitation of $325 \mathrm{~nm}$ and total testosterone concentration (TT) in serum was found for all serum samples $(r=-0.36, p=0.03)$. When only endurance-trained groups of rats are taken into account, this correlation becomes stronger $(r=-0.48, p=0.045)$. It means that a decrease in fluorescence at ex. $325 \mathrm{~nm}$ could indicate testosterone abuse. The fluorescence intensity at excitation of $365 \mathrm{~nm}$ shows the negative correlation $(r=-0.35)$ with FFA level in sedentary rats, which becomes weaker $(r=-0.23)$ when the endurance-trained rats are included into analysis. It follows that the strength of correlation depends on use it or not endurance training. Such behavior seems to be justified because, as reported by Newsholme and Blömstrand [23], physical activity may elevate plasma level of FFAs. Moreover Langfort et al. [24] reported that testosterone affects hormonesensitive lipase (HSL) and enhances lipolysis.

\section{Final remarks}

Our studies prove that the effects of endurance training and administration of testosterone are reflected in the fluorescence spectra of rat sera. Changes in fluorescence originating from metabolites in the visible region 
are more substantial than in the ultraviolet region dominated by Trp emission. The opposite effects of endurance training and administration of a lower dose of TE has been observed in fluorescence measurements. The high dose of TE blunts the differences in fluorescence of sera collected from trained and sedentary rats, probably due to steroid over-dose. The actual interpretation of these interesting results needs to be validated in human studies. Fluorescence spectroscopy seems to be a promising method for estimating the effects of endurance training and the usage of AAS in health care and sport settings.

\section{Acknowledgments}

Supported by statutory funds from the University of Silesia and from the Jerzy Kukuczka Academy of Physical Education.

\section{References}

[1] F. Hartgens, H. Kuipers, Sports Med. 34, 513 (2004).

[2] E.L. Cadore, L.F.M. Kruel, in: Sex Hormones, Ed. R. Dubey, InTech, Rijeka 2012, p. 277.

[3] A.S. Maior, C. Belchior, R.C. Sanches, T.O. da Silva, T. Leonelli, P.A. Schwingel, R. Simão, M. Marocolo, J.H.M. Nascimento, Int. J. Sport Exerc. Sci. 3, 27 (2011).

[4] S. Bhasin, L. Woodhouse, R. Casaburi, A.B. Singh, D. Bhasin, N. Berman, X. Chen, K.E. Yarasheski, L. Magliano, C. Dzekov, J. Dzekov, R. Bross, J. Phillips, I. Sinha-Hikim, R. Shen, T.W. Storer, Am. J. Physiol. Endocrinol. Metab. 281, E1172 (2001).

[5] A. Michnik, Z. Drzazga, E. Sadowska-Krępa, B. Kłapcińska, J. Therm. Anal. Calorim. 115, 2231 (2014).

[6] G.A. Wagnieres, W.M. Star, B.C. Wilson, Photochem. Photobiol. 68, 603 (1998).

[7] A.C. Croce, G. Bottiroli, Eur. J. Histochem. 58, 320 (2014).

[8] B.C. Wilson, in: Comprehensive Biomedical Physics, 1st ed., Vol. 10, Physical Medicine and Rehabilitation, Eds. A. Brahme, S. Zhou, L. Zhou, Elsevier, United Kingdom 2014, p. 205.
[9] Z. Drzazga, K. Michalik, K. Maciejewska, M. Kaszuba, B. Nowińska, H. Trzeciak, Photochem. Photobiol. 86, 87 (2010).

[10] Z. Drzazga, K. Maciejewska, K. Michalik, M. Kaszub, B. Nowińska, J. Fluoresc. 21, 883 (2011).

[11] O.S. Wolfbeis, M. Leiner, Anal. Chim. Acta 167, 203 (1985).

[12] M.J.P Leiner, R.J. Schaur, G. Desoye, O.S. Wolfbeis, Clin. Chem. 32, 1974 (1986).

[13] M.R. Hubmann, M.J.P. Leiner, R.J. Schaur, Clin. Chem. 36, 1880 (1990).

[14] L. Nørgaard, G. Soletormos, N. Harrit, M. Albrechtsen, O. Olsen, D. Nielsen, K. Kapmann, R. Bro, J. Chemometrics 21, 451 (2007).

[15] E. Sadowska-Krępa, B. Kłapcińska, S. Jagsz, A. Sobczak, S.J. Chrapusta, M. Chalimoniuk, P. Grieb, S. Poprzęcki, J. Langfort, Cardiovasc. Toxicol. 11, 118 (2011).

[16] G. Kanayama, J.I. Hudson, H.G. Jr. Pope, Drug Alcohol Depend 98, 1 (2008).

[17] J.R. Lakowicz, Principles of Fluorescence Spectroscopy, 3rd ed. Springer Science Business Media, LLC, New York 2006.

[18] E. Gianazza, I. Eberini, P. Villa, M. Fratelli, C. Pinna, R. Wait, M. Gemeiner, I. Miller, J. Chromatogr. B 771, 107 (2002).

[19] G. Münch, R. Keis, A. Wessels, P. Riederer, U. Bahner, A. Heidland, T. Niwa, H.D. Lemke, R. Schinzel, Eur. J. Clin. Chem. Clin. Biochem. 35, 669 (1997).

[20] M. Bohlooli, A.A. Moosavi-Movahedi, F. Taghavi, A.A. Saboury, P. Maghami, A. Seyedarabi, F. Moosavi-Movahedi, F. Ahmad, A. Shockravi, M. Habibi-Rezaei, Mol. Biol. Rep. 41, 3705 (2014).

[21] S. Fujiwara, A. Amisaki, Biochim. Biophys. Acta 1830, 5427 (2013)

[22] J. Hodosy, D. Ostatníková, M. Cagánová, M. Kovácsová, M. Mikulajová, L. Guller, Z. Putz, P. Celec, Pharmacol. Biochem. Behav. 101, 85 (2012).

[23] E.A. Newsholme, E. Blömstrand, J. Nutr. 136, 274S (2006).

[24] J. Langfort, S. Jagsz, P. Dobrzyn, Z. Brzezinska, B. Klapcinska, H. Galbo, J. Gorski, Biochem. Biophys. Res. Commun. 399, 670 (2010). 